\title{
An application on the second-order generalized difference equations
}

\author{
Mariasebastin Maria Susai Manuel ${ }^{1}$, Adem Kılıçman² ${ }^{2}$, Gnanadhass Britto Antony Xavier ${ }^{3}$, \\ Rajan Pugalarasu ${ }^{3}$ and Devadanam Suseela Dilip ${ }^{3}$
}

\author{
"Correspondence: \\ akilicman@putra.upm.edu.my \\ ${ }^{2}$ Department of Mathematics and \\ Institute for Mathematical Research, \\ University Putra Malaysia, UPM, \\ Serdang, Selangor 43400, Malaysia \\ Full list of author information is \\ available at the end of the article
}

\begin{abstract}
In this paper, we study the solutions of the second-order generalized difference equation having the form of

$$
\Delta_{\ell}^{2} u(k)+f(k, u(k))=0, \quad k \in[a, \infty), a>0, \ell \in(0, \infty),
$$

where $\Delta_{\ell} u(k)=u(k+\ell)-u(k)$. Then we provide applications on $\ell_{2(\ell)}$ and $c_{0(\ell)}$.

AMS Subject Classification: 39A12; 39A70; 47B39; 39B60

Keywords: generalized difference equation; generalized difference operator; finite series and infinite series
\end{abstract}

\section{Introduction and preliminaries}

The basic theory of difference equations is based on the difference operator $\Delta$ defined as $\Delta u(k)=u(k+1)-u(k), k \in \mathbb{N}=\{0,1,2,3, \ldots\}$, which allows the recursive computation of solutions. Later, the following definition was suggested for $\Delta_{\ell}$ by [1-3] and [4]:

$$
\Delta_{\ell} u(k)=u(k+\ell)-u(k), \quad k \in \mathbb{R}, \ell \in \mathbb{R}-\{0\} ;
$$

however, no significant progress took place on this line. Recently, equation (2) was reconsidered and its inverse was defined by $\Delta_{\ell}^{-1}$, and many interesting results in applications such as in number theory as well as in fluid dynamics were obtained; see, for example, [5]. By extending the study for sequences of complex numbers and $\ell$ to be real, some new qualitative properties like rotatory, expanding, shrinking, spiral and weblike were studied for the solutions of difference equations involving $\Delta_{\ell}$. The $\ell_{2}$ and $c_{0}$ solutions of the secondorder difference equation of (1) when $\ell=1$ were discussed in [6] and further generalized in [7]. In this paper, we discuss some applications of $\Delta_{\ell}$ in the finite and infinite series of number theory.

In this section, we present some of the preliminary definitions and results which will be useful for future discussion. The following definitions were held in [5] and [8], respectively.

Definition 1.1 Let $u(k), k \in[0, \infty)$, be a real- or complex-valued function and $\ell \in(0, \infty)$. Then the generalized difference operator $\Delta_{\ell}$ is defined as

$$
\Delta_{\ell} u(k)=u(k+\ell)-u(k) .
$$

@ 2013 Manuel et al.; licensee Springer. This is an Open Access article distributed under the terms of the Creative Commons Attribution License (http://creativecommons.org/licenses/by/2.0), which permits unrestricted use, distribution, and reproduction in any medium, provided the original work is properly cited. 
Then the inverse of $\Delta_{\ell}$ denoted by $\Delta_{\ell}^{-1}$ is defined as follows: If

$$
\Delta_{\ell} v(k)=u(k), \quad \text { then } v(k)=\Delta_{\ell}^{-1} u(k)+c_{j},
$$

where $c_{j}$ is a constant for all $k \in \mathbb{N}_{\ell}(j), j=k-\left[\frac{k}{\ell}\right] \ell$. If $\lim _{k \rightarrow \infty} u(k)=0$, then we can take $c_{j}=0$. Further, the generalized polynomial factorial for $\ell>0$ is defined as

$$
k_{\ell}^{(n)}=k(k-\ell)(k-2 \ell) \cdots(k-(n-1) \ell) .
$$

The following lemmas were proved in [9] and [10], respectively.

Lemma 1.2 (Product formula) Let $u(k)$ and $v(k), k \in[0, \infty)$, be any two real-valued functions. Then

$$
\begin{aligned}
\Delta_{\ell}\{u(k) v(k)\} & =u(k+\ell) \Delta_{\ell} v(k)+v(k) \Delta_{\ell} u(k) \\
& =v(k+\ell) \Delta_{\ell} u(k)+u(k) \Delta_{\ell} v(k) .
\end{aligned}
$$

Lemma 1.3 Let $\ell>0, n \in \mathbb{N}(2), k \in(\ell, \infty)$ and $k_{\ell}^{(n)} \neq 0$. Then

$$
\Delta_{\ell}^{-1} \frac{1}{k_{\ell}^{(n)}}=\frac{-1}{(n-1) \ell(k-\ell)_{\ell}^{(n-1)}}+c_{j} .
$$

Definition 1.4 A function $u(k), k \in[a, \infty)$, is said to be in the $\ell_{2(\ell)}$-space if

$$
\sum_{\gamma=0}^{\infty}|u(a+j+\gamma \ell)|^{2}<\infty \quad \text { for all } j \in[0, \ell)
$$

If $\lim _{r \rightarrow \infty}|u(a+j+r \ell)|=0$ for all $j \in[0, \ell)$, then $u(k)$ is said to be in the $c_{0(\ell)}$-space.

In what follows, we have the summation formula for finite and infinite series.

Lemma 1.5 If a real-valued function $u(k)$ is defined for all $k \in[0, \infty)$, then

$$
\Delta_{\ell}^{-1} u(k)=\sum_{r=1}^{\left[\frac{k}{\ell}\right]} u(k-r \ell)+c_{j}
$$

where $c_{j}$ is a constant for all $k \in \mathbb{N}_{\ell}(j), j=k-\left[\frac{k}{\ell}\right] \ell$. Since $[0, \infty)=\bigcup_{0 \leq j<\ell} N_{\ell}(j)$, each complex number $c_{j}(0 \leq j<\ell)$ is called an initial value of $k \in N_{\ell}(j)$. Usually, each initial value $c_{j}$ is taken from any one of the values $u(j), u(j+\ell), u(j+2 \ell)$, etc. Further, if $\lim _{k \rightarrow \infty} u(k)=0$ and $\ell>0$, then

$$
\Delta_{\ell}^{-1} u(k)=-\sum_{r=0}^{\infty} u(k+r \ell) .
$$

Proof Assume $z(k)=\sum_{r=0}^{\infty} u(k+r \ell)$. Then

$$
\Delta_{\ell} z(k)=\sum_{r=0}^{\infty} u(k+\ell+r \ell)-\sum_{r=0}^{\infty} u(k+r \ell)=-u(k) .
$$

Now, the proof follows from $\lim _{k \rightarrow \infty} u(k)=0$ and Definition 1.1. 
The next lemma is an expansion of Lemma 1.5 and its proof is straightforward.

Lemma 1.6 If $\lim _{k \rightarrow \infty} u(k)=0$ and $\ell>0$, then

$$
\Delta_{\ell}^{-2} u(k)=\sum_{r_{1}=0}^{\infty} \sum_{r_{2}=0}^{\infty} u\left(k+r_{1} \ell+r_{2} \ell\right)
$$

Theorem 1.7 Let $n \in \mathbb{N}(2), k \in(0, \infty)$ such that $k_{\ell}^{(n)} \neq 0$. Then

$$
\sum_{r=0}^{\infty} \frac{1}{(k+r \ell)_{\ell}^{(n)}}=\frac{1}{(n-1) \ell(k-\ell)_{\ell}^{(n-1)}} .
$$

Proof The proof follows from Lemma 1.3 and Lemma 1.5 by taking $u(k)=\frac{1}{k_{\ell}^{(n)}}$ and $c_{j}=0$.

Corollary 1.8 Let $k \in(\ell, \infty)$ and $\ell \in(0, \infty)$. Then

$$
\sum_{r=0}^{\infty} \frac{1}{(k+r \ell)(k+r \ell-\ell)}=\frac{1}{\ell(k-\ell)}
$$

Proof Since $\Delta_{\ell}^{-1} \frac{1}{k(k-\ell)}=\frac{-1}{\ell(k-\ell)}$, the proof follows from Theorem 1.7 by taking $n=2$.

\section{Applications of $\Delta_{\ell}$ in number theory}

In this section, we present some formulae and examples to find the values of finite and infinite series in number theory as an application of $\Delta_{\ell}$. The following theorem and example were given in [7]. In fact, the example is to illustrate Theorem 2.1.

Theorem 2.1 Let $k \in[\ell, \infty)$ and $\ell \in(0, \infty)$. Then

$$
\sum_{r=1}^{\left[\frac{k}{\ell}\right]+s} \frac{(k-r \ell+2 \ell)^{2}-3 \ell^{2}}{\ell^{r}(k-r \ell+4 \ell)_{\ell}^{(2)}(k-r \ell+\ell)_{\ell}^{\left(\left\lceil\frac{k-r \ell+\ell}{\ell}\right\rceil\right)}}=\frac{c_{j}}{\ell^{\left\lceil\frac{k}{\ell}\right\rceil}}-\frac{1}{(k+3 \ell) k_{\ell}^{\left(\left\lceil\frac{k}{\ell}\right\rceil\right)}}
$$

where $s=-1$ for $k \in \mathbb{N}_{\ell}(\ell), s=0$ for $k \notin \mathbb{N}_{\ell}(\ell)$ and each $c_{j}$ is a constant for all $k \in \mathbb{N}_{\ell}(j)$, $j=k-\left[\frac{k}{\ell}\right] \ell$. In particular $c_{j}$ is obtained from (14) by substituting $k=\ell+j$.

Example 2.2 By taking $\ell=1.7, k=2$ and $j=0.3$ in (14), we get $c_{j}=\frac{85}{81}$ and hence (14) becomes

$$
\begin{aligned}
& \sum_{r=1}^{\left[\frac{k}{\ell}\right]} \frac{(k-1.7 r+2(1.7))^{2}-3(1.7)^{2}}{1.7 r(k-1.7 r+4(1.7))_{1.7}^{(2)}(k-1.7 r+1.7)_{1.7}^{\left.\left(\frac{k-1.7 r+1.7}{1.7}\right\rceil\right)}} \\
& \quad=\frac{85}{81(1.7)^{\left\lceil\frac{k}{1.7}\right\rceil}}-\frac{1}{(k+3(1.7)) k_{1.7}^{\left(\left\lceil\frac{k}{1.7}\right]\right)}}, \quad k=2,3.7,5.4, \ldots
\end{aligned}
$$

Theorem 2.3 Let $k \in[\ell, \infty)$ and $\ell \in(0, \infty)$. Then

$$
\sum_{r=0}^{\infty} \frac{k+r \ell+\ell}{3^{\frac{k+r \ell}{\ell}}(2(k+r \ell)+\ell)_{2 \ell}^{(2)}}=\frac{1}{4(3)^{\frac{k}{\ell}-1}(2 k-\ell)}
$$


Proof By Definition 1.1, we find

$$
\Delta_{\ell}^{-1}\left(\frac{(k+\ell)}{3^{\frac{k}{\ell}}(2 k+\ell)_{2 \ell}^{(2)} k}\right)=\frac{-1}{4(3)^{\frac{k}{\ell}-1}(2 k-\ell)}
$$

and (15) follows by Lemma 1.5 and $c_{j}=0$ as $k \rightarrow \infty$.

The following is the illustration for Theorem 2.3.

Example 2.4 Taking $\ell=0.2$ in (15), we arrive at

$$
\frac{k+0.2}{3^{\frac{k}{0.2}}(2 k+0.2)_{0.4}^{(2)}}+\frac{k+0.4}{3^{\frac{k+0.2}{0.2}}(2 k+0.6)_{0.4}^{(2)}}+\frac{(k+0.6)}{3^{\frac{k+0.4}{0.2}}(2 k+1)_{0.4}^{(2)}}+\cdots=\frac{1}{4(3)^{\frac{k}{0.2}-1}(2 k-0.2)}
$$

and one can take any value $k \in[\ell, \infty)$.

Theorem 2.5 For $k \in[\ell, \infty)$ and $\ell \in(0, \infty)$, then

$$
\sum_{r=0}^{\infty} \frac{(k+r \ell)^{3}-\ell^{3}}{\ell^{r}\left((k+r \ell)^{2}-2 \ell^{2}\right)_{\ell}^{(2)}(k+r \ell+\ell)_{\ell}^{\left(\left\lceil\frac{k+r \ell+\ell}{\ell}\right]\right)}}=\frac{1}{\left((k-\ell)^{2}-2 \ell^{2}\right) k_{\ell}^{\left(\left\lceil\frac{k}{\ell}\right\rceil\right)}} .
$$

Proof By Definition 1.1, we find

$$
\Delta_{\ell}^{-1} \frac{\left(k^{3}-\ell^{3}\right) \ell^{\left\lceil\frac{k}{\ell}\right\rceil}}{\left(k^{2}-2 \ell^{2}\right)_{\ell}^{(2)}(k+\ell)_{\ell}^{\left(\left\lceil\frac{k+\ell}{\ell}\right\rceil\right)}}=\frac{-\ell^{\left\lceil\frac{k}{\ell}\right\rceil}}{\left((k-\ell)^{2}-2 \ell^{2}\right) k_{\ell}^{\left(\left\lceil\frac{k}{\ell}\right\rceil\right)}}
$$

and (16) follows by (10) and $c_{j}=0$ as $k \rightarrow \infty$.

The following theorem generates the formula to find the sum of first partial sums of an infinite series.

Theorem 2.6 For the positive integer $n \in \mathbb{N}(3), k \in[2 \ell, \infty)$ and $\ell \in(0, \infty)$,

$$
\sum_{r_{2}=0}^{\infty} \sum_{r_{1}=0}^{\infty} \frac{1}{\left(k+r_{2} \ell+r_{1} \ell\right)_{\ell}^{(n)}}=\frac{1}{(n-1)(n-2) \ell^{2}(k-2 \ell)_{\ell}^{(n-2)}}
$$

Proof Using Definition 1.1 and operating $\Delta_{\ell}^{-1}$ on (7), we find

$$
\Delta_{\ell}^{-2} \frac{1}{k_{\ell}^{(n)}}=\frac{1}{(n-1)(n-2) \ell^{2}(k-2 \ell)_{\ell}^{(n-2)}}
$$

and hence (17) follows by Lemma 1.6 as $c_{j}=0$ when $k \rightarrow \infty$.

The following example illustrates Theorem 2.6.

Example 2.7 Substituting $\ell=0.5, n=4$ in (17), we obtain

$$
\frac{1}{(k)_{0.5}^{(4)}}+\frac{2}{(k+0.5)_{0.5}^{(4)}}+\frac{3}{(k+1)_{0.5}^{(4)}}+\cdots=\frac{1}{6(0.5)^{2}(k-1)_{0.5}^{(2)}} .
$$


In particular, when $k=2$, the above series becomes

$$
\frac{1}{2 \times 1.5 \times 1 \times 0.5}+\frac{2}{2.5 \times 2 \times 1.5 \times 1}+\frac{3}{3 \times 2.5 \times 2 \times 1.5}+\cdots=\frac{1}{6 \times 0.5^{3}} .
$$

Similarly, one can take any value for $k \in[2 \ell, \infty)$ and $\ell \in(0, \infty)$.

The following example shows that $\frac{1}{k_{\ell}^{(n)}} \in c_{0(\ell)}$ and $\ell_{2(\ell)}$ when $k_{\ell}^{(n)} \neq 0$.

Example 2.8 Let $n \in \mathbb{N}(2), \ell \in(0, \infty), j \in[0, \ell)$ and $a \geq n \ell$. Replacing $k$ by $a+j$, in (12), we get

$$
\sum_{r=0}^{\infty} \frac{1}{(a+j+r \ell)_{\ell}^{(n)}}=\frac{1}{(n-1) \ell(a+j-\ell)_{\ell}^{(n-1)}} .
$$

Since

$$
\left|\frac{1}{(a+j+r \ell)_{\ell}^{(n)}}\right|^{2}<\frac{1}{(a+j+r \ell)_{\ell}^{(n)}}
$$

thus from (18) it follows that

$$
\sum_{r=0}^{\infty}\left|\frac{1}{(a+j+r \ell)_{\ell}^{(n)}}\right|^{2}<\sum_{r=0}^{\infty} \frac{1}{(a+j+r \ell)_{\ell}^{(n)}}=\frac{1}{(n-1) \ell(a+j-\ell)_{\ell}^{(n-1)}}<\infty .
$$

The function $\frac{1}{k_{\ell}^{(n)}} \in \ell_{2(\ell)}$ follows from Definition 1.4 by taking

$$
u(a+j+r \ell)=\frac{1}{(a+j+r \ell)_{\ell}^{(n)}} .
$$

Since $\lim _{r \rightarrow \infty} \frac{1}{(a+j+r \ell)_{\ell}^{(n)}}=0$ for all $j \in[0, \ell)$, Definition 1.4 yields $\frac{1}{k_{\ell}^{(n)}} \in c_{0(\ell)}$.

\section{Concluding remarks}

In the present work, we provide an application on $\ell_{2(\ell)}$ and $c_{0(\ell)}$ and solutions of the second-order some generalized difference equation.

\section{Competing interests}

The authors declare that they have no competing interests.

\section{Authors' contributions}

All authors contributed equally to the manuscript and typed, read, and approved the final manuscript.

\section{Author details}

'Department of Science and Humanities, R.M.D. Engineering College, Kavaraipettai, Tamil Nadu 601206, India.

${ }^{2}$ Department of Mathematics and Institute for Mathematical Research, University Putra Malaysia, UPM, Serdang, Selangor 43400, Malaysia. ${ }^{3}$ Department of Mathematics, Sacred Heart College, Vellore District, Tirupattur, Tamil Nadu 635601, India.

\section{Acknowledgements}

The authors express their sincere gratitude to the referees for their valuable suggestions and comments. The second author also acknowledges that this project was partially supported by University Putra Malaysia under the ERGS Grant Scheme having project number 5527068. 


\section{References}

1. Agarwal, RP: Difference Equations and Inequalities. Dekker, New York (2000)

2. Mickens, RE: Difference Equations. Van Nostrand-Reinhold, New York (1990)

3. Elaydi, SN: An Introduction to Difference Equations, 2nd edn. Springer, Berlin (1999)

4. Kelley, WG, Peterson, AC: Difference Equations. An Introduction with Applications. Academic Press, San Diego (1991)

5. Manuel, MMS, Xavier, GBA, Thandapani, E: Theory of generalized difference operator and its applications. Far East J. Math. Sci. 20(2), 163-171 (2006)

6. Popenda, J, Schmeidal, E: Some properties of solutions of difference equations. Fasc. Math. 13, 89-98 (1981)

7. Manuel, MMS, Kılıçman, A, Xavier, GBA, Pugalarasu, R, Dilip, DS: On the solutions of second order generalized difference equations. Adv. Differ. Equ. 2012, 105 (2012). doi:10.1186/1687-1847-2012-105

8. Ferreira, RAC, Torres, DFM: Fractional $h$-difference equations arising from the calculus of variations. Appl. Anal. Discrete Math. 5(1), 110-121 (2011). doi:10.2298/AADM110131002F

9. Manuel, MMS, Xavier, GBA, Dilip, DS, Chandrasekar, V: General partial sums of reciprocals of products of consecutive terms of arithmetic progression. Int. J. Comput. Appl. Math. 4(3), 259-272 (2009)

10. Manuel, MMS, Xavier, GBA: Generalized difference calculus of sequences of real and complex numbers. Int. J. Comput. Numer. Anal. Appl. 6(4), 401-415 (2004)

doi:10.1186/1687-1847-2013-35

Cite this article as: Manuel et al.: An application on the second-order generalized difference equations. Advances in Difference Equations 2013 2013:35.

\section{Submit your manuscript to a SpringerOpen ${ }^{0}$ journal and benefit from:}

- Convenient online submission

- Rigorous peer review

- Immediate publication on acceptance

- Open access: articles freely available online

- High visibility within the field

- Retaining the copyright to your article 